\title{
EFFICIENT AND PREDICTABLE CO-OPERATIVE MULTICAST ROUTING WITH DEFENSIVE MECHANISM IN MANETS
}

\author{
Dilip S $S^{1}$ T. R. Mahesh ${ }^{2}$ \\ ${ }^{I}$ Mtech Student, Department of Computer Science \& Engineering T John Institute of technology, Karnataka, India \\ ${ }^{2}$ Professor and Head, Department of Computer Science \& Engineering T John Institute of technology, Karnataka, \\ India
}

\begin{abstract}
MANET-Mobile Adhoc System is a self-arranging system of portable hubs in which they are associated by remote. This paper is proposed for the most part to diminish the variables, for example, End to End delay, value, power utilization, system burden, and bundle misfortune.In this proposal we are using CM-AODV Cooperative Multicast[7] - Adhoc on Demand Distance Vector as a cooperative directing convention.NS-2 is used for the reenactment reasonwhere the packet loss in the mobile adhoc network are investigated through simulation [1]. Detective and Defensive mechanism is used to overcome the different routing attacks such as packet drop and grey holeattack [2]. We are utilizing CM-AODV Steering convention whose performance are compared to the existing AODV-Adhocon Demand Distance Vector Routing Protocol. The results Show that AODV loss more number of packetscompared to CM-AODV.For AODV more number of packets are related to congestion or the traffic related. Test information determines the parcel misfortune dispersion as the time is blasting which makes convention Poisson system unacceptable for displaying it. Preparatory results show self - comparable example that persuades that fractal model is promising to depict bundle misfortune in specially appointed systems. This work determine the rules for the outline of directing and stream control calculations and bits of knowledge in picking appropriate parameters in future reproduction and logical studies. Finally from the X-graph we can conclude that CM-AODV is more efficient and Reliable compared to the AODV and DSR Routing protocol[4].
\end{abstract}

Keywords: CM- AODV, Routing Protocol[4], Routing Attacks[2],NS-2[1]etc...

\section{INRTODUCTION}

MANETs -Mobile Adhoc Networks, here the nodes are continuously self-configured. The nodes are communicate with each other without any centralized base station and they are connected by wireless. The CMAODV is a reactive protocol, where path is established whenever the node wants to communicate with other node. This protocol consists of dynamic nodes (mobility)[8]. The target is to discover the path, identify the destination and deliver the encrypted packets between the source and destination. This can be seen through simulation, which can model real-life objectson a computer so that it can be studied. It can be also applicable to different science, engineering, or other application fields for various purposes .Here NS-2 is used for simulation purpose, NS2 plays a role of both simulator and emulator [6].The TCL debugger is utilized to troubleshoot TCL scripts, which gets to be important in the event that one is utilizing substantial scripts to control a recreation. It last comprises of three sorts of discrete occasion schedulers: pile, rundown, and hash-based timetable [1]. NS2 gives default usage to network hubs, joins between hubs, steering calculations, some vehicle level conventions particularly UDP and TCP and some of movement generators [1]. Likewise it contains some valuable administrations like the reenactment situation generator TCL debugger, and recreation topology generator.
EXISTISG PAPERS- MANETs faces various kinds of routing attacks[2] no specified node failure detection mechanism. Existing papers causes more routing overhead and packet loss.

The limitations of the existing system are as follows:

1. Specific Route Failure Detection Mechanism is required to each and every routing attack.

2. Increased cost, Network load and the packet loss has to be decreased.

3. Additional memory for running the node detection algorithm is to be required.

4. Due to the routing overhead life time of the node while processing the control packets get reduced.

The psychological feature of this concept is to design route processor for overcoming the limitations of exiting method. The mobile adhocnetworks have limited memory, life time, and Computational capabilities. Limitations of mobile nodes have to be considered while proposing a solution in hardware. Hence, the main idea is to reduce the packet loss and achieve higher throughput. The Execution correlation of AODV and proposed CMAODV directing conventions are investigated and tried when recreations are performed on NS-2 test system. The CMAODV directing convention is utilized to beat the issues in the current technique. End-toend postpone and cost will get lessen likewise it decrease 
the system burden and parcel misfortune. Exceptional fixation is given for controlling as far as possible. The AODV-Specially appointed On-interest Separation Vector Convention is a Receptive steering Convention. That comprises of both the elements of Proactive and Receptive steering i.e. DSDV -Destination Succession Separation Vector and DSR-Dynamic Source Directing separately. The expense of the CM-AODV get diminished contrasted with AODV [4]. The quantity of hubs used for the each and every exchange from allocated source hub to destination hub will get decreased.

\section{PROPOSED WORK}

MANETs network concentrates mainly on remote communication without centralized base station. Remote correspondence has different application in various security levels .Prior there was just a settled remote correspondence. System exists where correspondence reach is get restricted. Presently Propelled Versatile Specially appointed system are presented where all hubs offer information among themselves. Each specially appointed steering convention have diverse directing level components, for example, System load, End to End Delay, bundle conveyance proportion and power utilization gets fluctuated. In CMAODV the hubs can build up or interface with other hub and leave the system at whatever point they required. Directing for the most part manages the course revelation between the source and destination in a secured way. According to the necessity of framework Hubs the system topology may fluctuate for each specific interims of time. The directing Conventions are fundamentally separated into Responsive and Proactive Steering Convention. Proactive directing conventions are table driven, full or halfway duplicate of the system topology and expenses are kept up by Connection state calculations for all known connections. Receptive directing convention (CMAODV) makes and keeps up courses just if these are required, i.e., the courses are built up on interest. Typically they use on interest separation vector steering calculations that keep just data about next hub to contiguous neighbours and expenses for ways to every single known destination. Consequently, interface state directing calculations use less transmission capacity, more dependable. CMAODV directing convention is a responsive steering convention. It use the Dijkstra's out of reach vector count to find the briefest way. In this a path to an end is determined. When a centre point needs to send a bundle to that goal destination. Courses are kept up the length of they are required by the source need to send bundles. As of right now when pack is shown from source to destination there are different centres included among the productive transmissions of packages. CM-ADOV controlling tradition uses Course Ask for (RREQ) Course Answer (RREP) and Course Blunder (RERR) as a control signal for the correspondence reason. Right when a source centre point need to make an impact on any destination centre point and if it doesn't have an affirmed course to that destination it looks for a course to locate the other centre. Source centre point sends a RREQ group to its neighbours, which then forward the requesting to their neighbours, it is continued until it accomplishes the destination.

While preparing the course ask for middle of the road hubs get stacked to its particular steering table which incorporate the location of the neighbors from which the primary duplicate of the show bundle is gotten. This will give to discover a way. In the event that if there should be an occurrence of some extra duplicates of same Course ask for arrived then the parcels get tossed. At the point when the RREQ achieves the objective destination hub, the destination or middle of the road hub reacts through directing a RREP-Course answer parcel toward neighbor from which it initially got the RREQ. It is seen that because of awful linkage quality and absence of legitimate correspondence channel between the hubs parcel may get dropped. At times correspondence is fruitful however the backend variables, for example, Force utilization End to End delay, Directing overhead and bounce constrain truly makes arrange truly expensive and questionable one. In CMAODV directing table assumes the imperative part to beat these circumstances. It incorporates the section for every hub with the data in regards to the succession number for IP location of destination hub. The Course ask for, Course answer and course blunder charges are gotten for overhauling of the succession number of the hub. The end center point can uplift its categorizing number when there is the perfect open door for source center point to start a course look for or when there is the perfect open door for destination center point to deliver the Course answer message against the Course request response of witness center. In controlling table the course gets upgraded with new game plan of numbers when it is higher than the end gathering numbers. In the event that the source not get the answer or if the way to the destination is invalid, it resends the RREQ and at same time in reverse course to the source is made. There are other two methodologies, the first is the point at which the new arrangement number and destination succession numbers are equivalent however in the event that total number of jump in addition to one extra one bounce in new grouping steering table is not as much as bounce check in the current destination succession number and besides when the current succession number is obscure. In the event that any of the hub gets influenced or if any next jump get fizzled then the Course mistake message sent to the source and the source again send with course ask for message. Once the Source get mistake message then it picks an option way through the agreeable hubs in sending the parcels.

\section{DESIGN}

When the source node wants to reach the destination not having any specified path then the source hub surges a 
course demand to its neighboring hubs in a system. The Course ask.is broadcasted to all over the network and it finally it reaches the destination. Based on the source address the destination node finds the shortest path using the shortest path routing algorithm and it sends the Route Reply along the path. Every selected node acts as a root node and activates its co-operative nodes. Data packets are sent through the cooperative nodes if any failure of the root node occurs. Monitoring node in a network uses detective and defensive mechanism to identify the affected nodes in a network and enable the source to restrict the use of affected nodes and choose the secured path along the cooperative nodes [7].

Network simulator NS2 is used for simulation purpose and it is used for predicting thebehaviour of the network. Various attributes of the network has been modelled environment to access the network under different situations. In the versatile adhoc systems is normally displayed with gadgets, test systems, movement and so forth and its execution are resolved. Commonly, clients can modify the test system to satisfy their particular investigation needs. Contrasted with that of expense and time included in setting up a whole proving ground containing switches and information connects, numerous organized PCs, system test systems are moderately quick and modest. This permits system designers, scientists to test the conditions that may be especially troublesome or costly to copy them utilizing genuine equipment. In CM-AODV first it chooses a node discovery phase it include configuration of nodes, transmission power and the receiving power. After configuration the nodes move to their respective position in a network which is shown in figure 1.

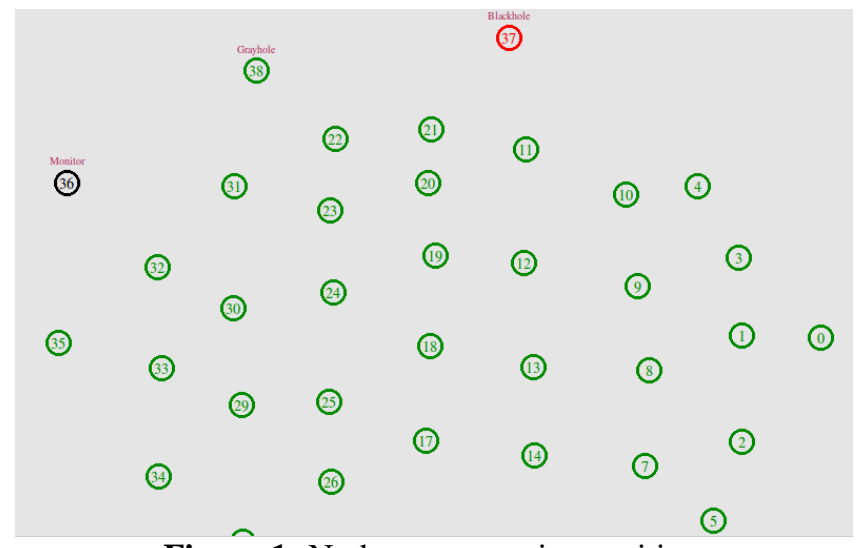

Figure 1: Nodes at respective position

In the above figure node 36 acts as a monitoring node. Node 37 and 38 are the infected nodes which causes routing attacks.

The source node 0 surges a course demand to its neighboring hubs and achieves the destination.

Based on source address the destination node chooses the shortest using shortest path algorithm and sends the route reply along the path to source node. Selected node acts as a root node and activates its cooperative node. Source node make use of the cooperative nodes in sending the data packets. Formation of cooperative nodes are shown in figure 2.

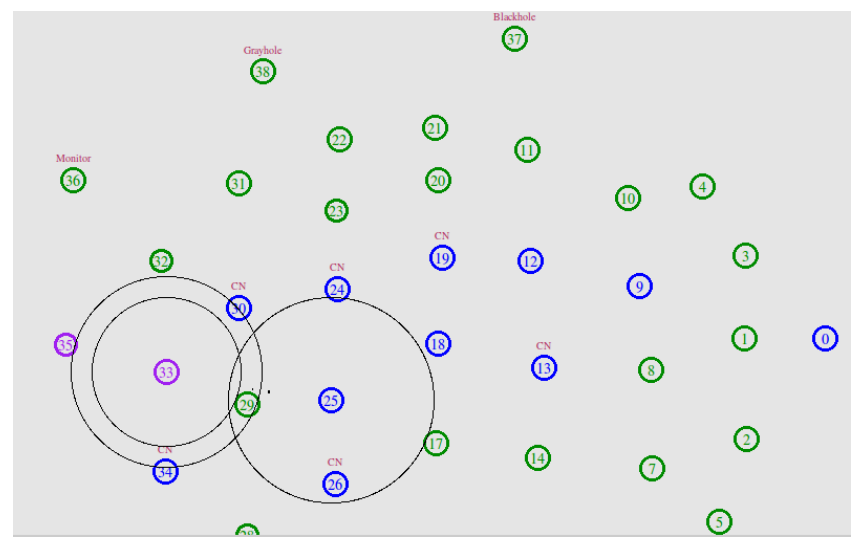

Figure 2: Formation of cooperative nodes

The root node 33 activate its cooperative node 30 and 34,the root node 25 activate its cooperative node 24 and 26, and the root node 18 activate its cooperative node 13 and 19 .

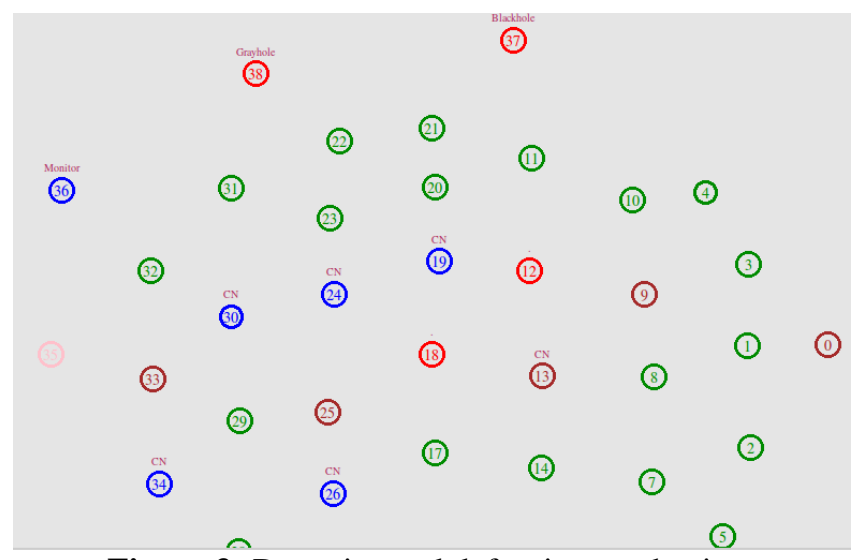

Figure 3: Detective and defensive mechanism

Node 37 and 38 causes a routing attack i.e. black hole and grey hole attack over the network. Node 36 acts as a monitoring node which identifies the nodes affected in a network and enable the source to restrict the use of affected nodes so that the source will send the data packets along the cooperative nodes. This kind of affected node detection and defensive mechanism is shown in figure 3 .

In the figure 3 , the monitoring node 36 identifies the malicious affected node12 and 18, after identifying the affected node it enable the source node to choose alternate path i.e. 0-9-13-25-33-35 along the cooperative nodes.

The flow chart shown below summarizes the action of a Cooperative multicasting in CMAODV protocol. 


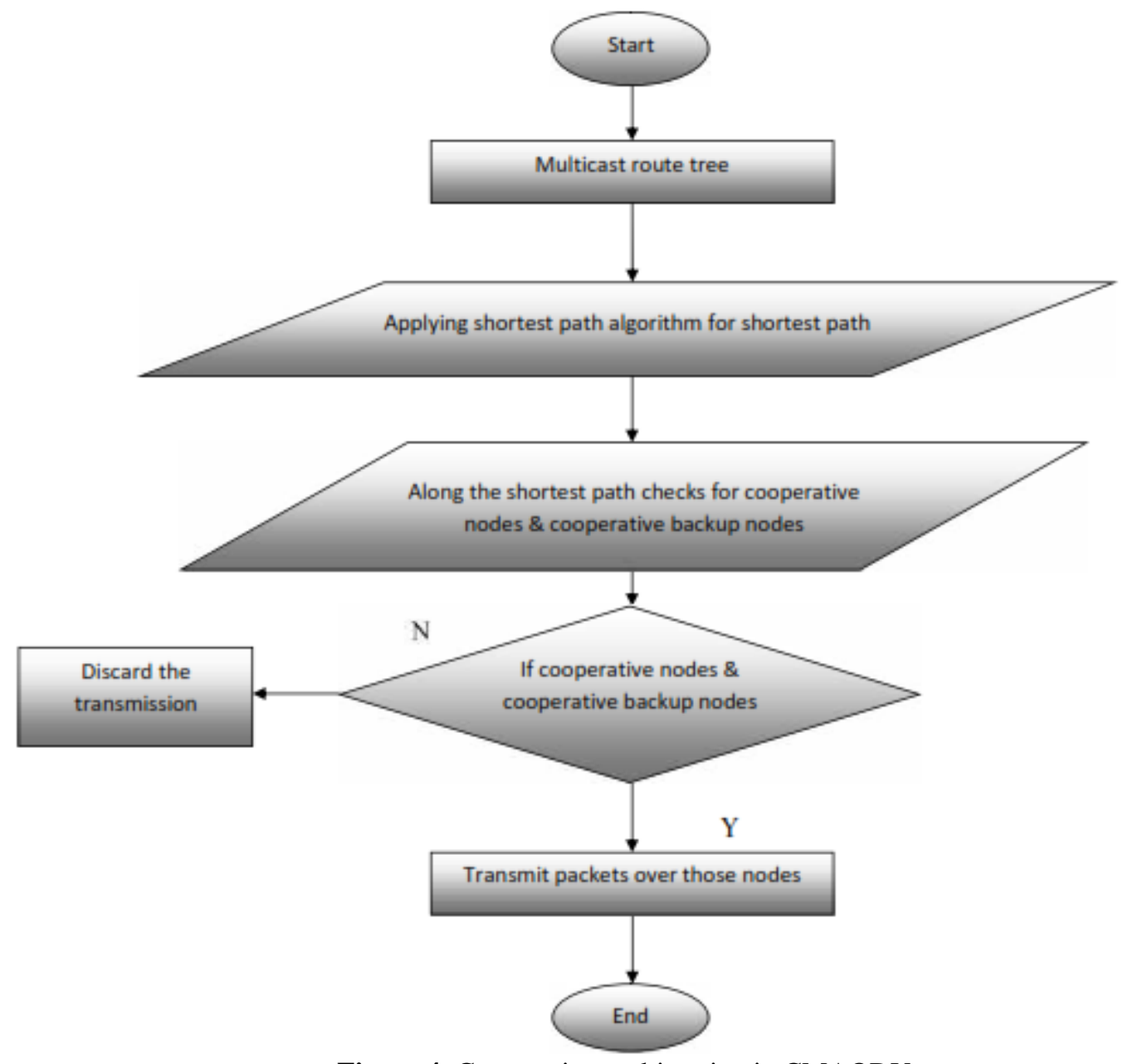

Figure 4: Cooperative multicasting in CMAODV

Step 1- Nodes in a network move to their respective position in a network position.

Step 2-source hub sends course demand to the hub to make course to destination.

Step3-Based on the source address destination node forms a multicast tree.

Step 4-Based on the information of multicast tree, destination node finds the shortest path using the shortest path algorithm.

Step 5- Formation of cooperative nodes.

Step 6- Transmission of data packets.

\section{SIMULATION CONSIDERATIONS}

Simulation done for 38 nodes in a network. Simulation done in an area of $1500 \mathrm{~mm} * 1000 \mathrm{~mm}$. Performance metrics such as packet loss comparison, throughput, and packet delivery ratio in CM-AODV routing protocol is compared to existing AODV-Adhoc On demand Distance Vector routing and DSR- Dynamic Source Routing.

The Reproduction Parameters are given underneath:

\begin{tabular}{|l|l|}
\hline Number of hubs & 38 \\
\hline Steering Convention & CM-AODV \\
\hline Movement Source & CBR \\
\hline Area & $1500 \mathrm{~mm} * 1000 \mathrm{~mm}$ \\
\hline Tool & NS-2.35 \\
\hline
\end{tabular}

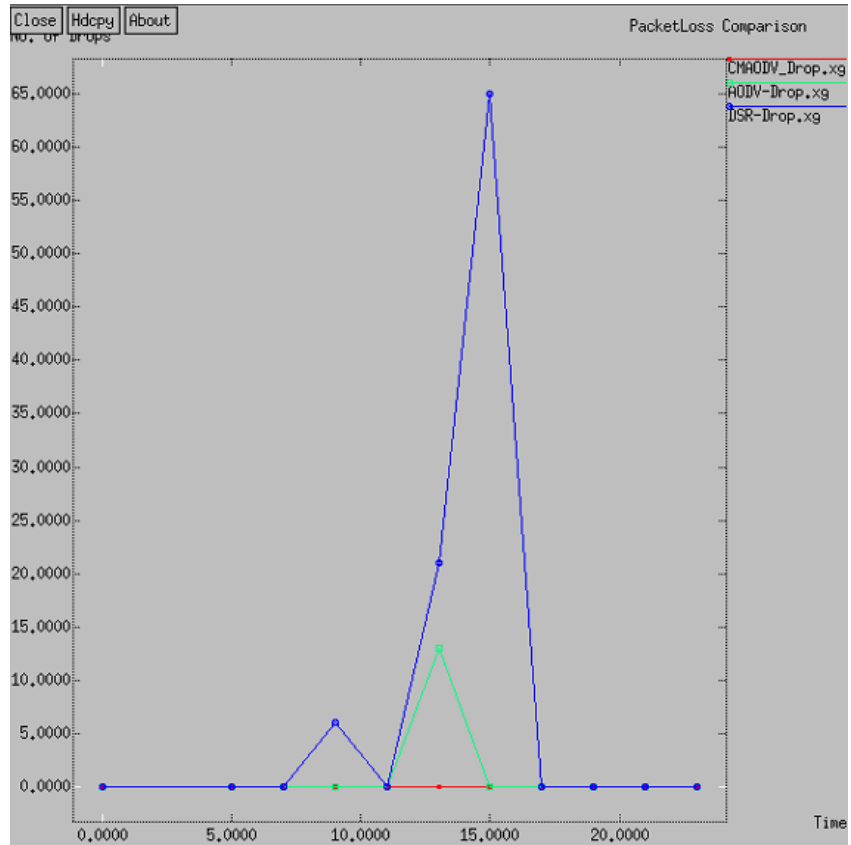

Figure 5: Packet Loss Comparison

Figure 5 indicates number of parcel drops is plotted against the time. In the chart just three information exchanges are considered. It is watched that DSR and AODV misfortunes more number of bundles contrasted with CM-AODV. 


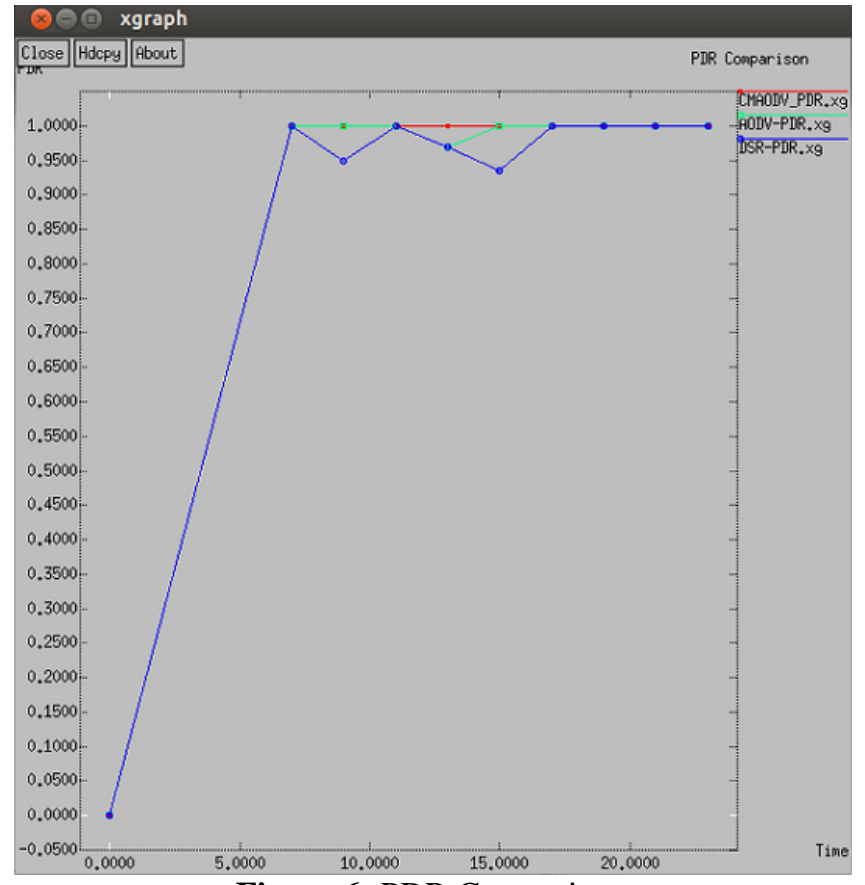

Figure 6: PDR Comparison

Figure 6 indicates number of PDR-Bundle Conveyance Proportion is plotted against the time. Here just three information exchanges are considered. It is watched that DSR and AODV conventions having less parcel conveyance proportion contrasted with CM-AODV.

Figure 7 demonstrates throughput examination diagram where throughput is plotted against the time. Here just three information exchanges are taken. It is watched that DSR and AODV conventions having throughput contrasted with CMAODV.

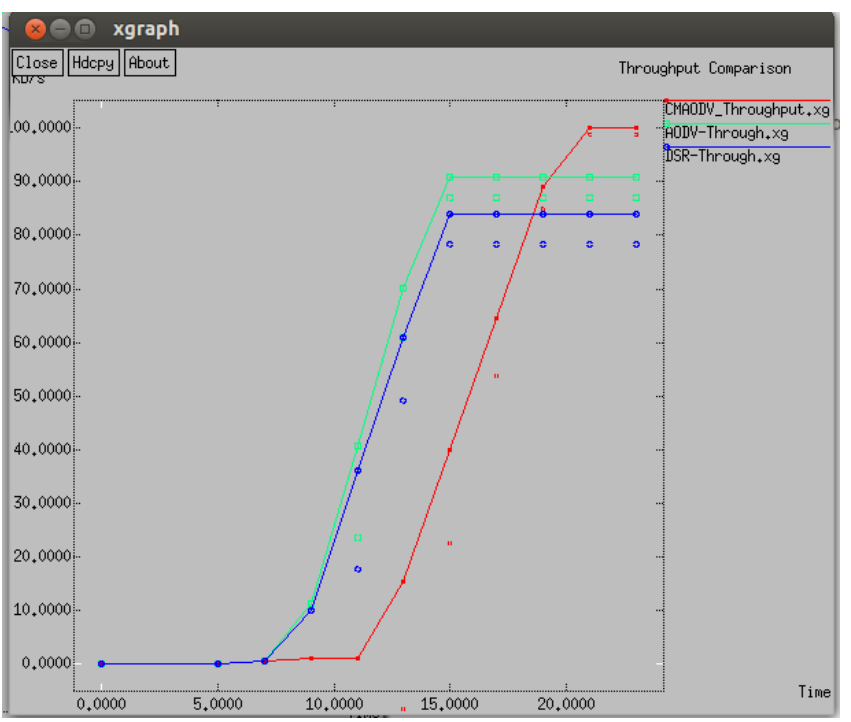

Figure 7: Throughput Comparison

\section{CONCLUSIONS}

From the $\mathrm{X}$-diagram we can reason that the execution measurements is productive and dependable in CM-AODV, for example, Parcel conveyance proportion, Bundle dropt, throughput, Parcel misfortune against time contrasted with existing DSR and AODV directing conventions with number of portable hubs of up to 38 utilizing NS-2.35 as a reproduction device. In the event that the system load happens CM-AODV is capable accomplish less bundles Drop when contrasted with existing AODV convention. Indeed, even the quantity of hubs is expanded, the CMAODV performs well and creates better throughput level and devours less vitality. In the event that the system load happens CM-AODV is capable accomplish less bundles Drop when contrasted with existing AODV convention.

\section{REFERENCES}

[1]. Mrs. SabaSiraj, Mr. Ajay Kumar Gupta, Mrs Rinku-Badgujar, "Network Simulation Tools Survey",International Journal of Advanced Research in Computer and Communication Engineering, Vol. 1, Issue 4, June 2012.

[2]. Manikandan, S.P. and R. Manimegalai, "Survey on mobile ad hoc network attacks andMitigation using routing protocols", American Journal of Applied Sciences, ISSN 1546-9239, 2012.

[3]. Sihai Zheng, Layuan Li and Yong Li, "A QoS Routing Protocol for Mobile Ad HocNetworks Based on Multipath", journal of networks, vol. 7, no. 4, April 2012.

[4]. Gupta N, Gupta R., "Routing Protocols in Mobile AdHocNetworks: an Overview", IEEE International Conference on Emerging Trends in Robotics and Communication, pp.173-177, 2010.

[5]. Neeraj Arya, Upendra Singh, Sushma Singh, "Detecting and avoiding of worm hole attack and collaborative blackhole attack on MANET using trusted AODV routing algorithm", Computer, Communication and Control (IC4), International Conference, ISBN- 978-1-47998163-2, 2015

[6]. E. Weingartner ; Distrib, H. vom Lehn , K. Wehrle, " A Performance Comparison of Recent Network Simulators", 2009 IEEE International Conference on Communications, E-ISBN : 978-1-4244-3435-0, 2009.

[7]. Arjun Chaudhary, SatvikSachdev, Tribikram Pradhan, SantoshKamath," Improving performance of MANET via cooperative communication with selective cooperation method considering dynamic traffic pattern using NS2", High Performance Computing and Applications (ICHPCA), International Conference,ISBN: 978-1-4799-5957-0, 2014. [8]. Amandeep Singh Bhatia, Rupinder Kaur Cheema, "Analysing and Implementing the Mobility over MANETS using Random Way Point Model “, International Journal of Computer Applications (0975 - 8887) Volume 68- No.17, April 2013. 